\section{In response}

Editor - We were interested to read the comments of Drs Kale and Handy and are pleased that our case report has generated interest and correspondence. We agree that hypoalbuminaemia is a potential cause of a metabolic alkalosis and, in retrospect, we should have included this information in the report. At $40 \mathrm{~g} / \mathrm{l}$ it was clearly not contributing to our patient's acid-base derangement.

While we acknowledge the interest of the correspondents, we feel they have misconstrued the message of our report. We did not suggest that administration of Hartmann's solution was the sole cause of our patient's severe alkalosis; the patient had symptoms of ionized hypocalcaemia prior to its administration. As both the abstract and the 'key learning points' box clearly state, we were using the case to illustrate various aspects of the diagnosis and treatment of normalcaemic tetany; one of which is that the administration of Hartmann's solution in this situation of volume contraction, alkalosis and paradoxical aciduria due to prevailing secondary hyperaldosteronism, was not appropriate. There seems to be little in the content of their argument that contradicts our conclusions.

In preparing our manuscript, we did not consider that Clinical Medicine was the correct forum for an in-depth metabolic discussion, but we would remind the correspondents that the hepatic metabolism of lactate consumes protons and generates bicarbonate. In a starving, volume-contracted patient with reduced renal perfusion, this will be the metabolic fate of the vast majority of infused lactate. As Hartmann himself demonstrated, 11 of 1/6 molar sodium lactate is potentially equivalent to $290 \mathrm{ml}$ of $5 \%$ sodium bicarbonate; non-metabolically orientated readers of the journal may find this easier to comprehend. Kale and Handy's reference to 'total body Hartmann's transfusion' is misleading and detracts from the message that administration of an alkalinizing fluid to an already symptomatically alkalotic patient is potentially hazardous.

Finally, it is well recognised that potassium deficiency and alkalosis cannot be

fully corrected unless there is satisfactory replenishment of chloride. The relevant chloride contents of Hartmann's solution and normal saline $(111 \mathrm{mEq} / \mathrm{l}$ and 154 $\mathrm{mEq} / \mathrm{l}$ respectively) provide a further reason (not included in our original report) that the latter would have been the most appropriate resuscitation fluid in this clinical scenario.

V SEHGAL $S$ VIJAYAN

$S$ YASMIN

U SRIRANGALINGAM WM DRAKE

Department of Endocrinology, St Bartholomew's Hospital, London

J PATI

Department of Urology, St Bartholomew's Hospital, London

\section{Cavitating pulmonary tuberculosis: a global challenge.}

Editor - We were concerned that a new treatment for tuberculosis (TB) was being advocated without the benefit of a randomised controlled trial (W Saeed, Clin Med February 2012 pp 40-1). The authors argue that cavitary pulmonary TB relapses following therapy in $21-25 \%$ of cases and that additional drugs (levofloxacin and amikacin) are required to prevent this. However, in a treatment trial of sputum smear-positive TB (a sine qua non for cavitary disease) with relatives supervising therapy in the follow-up phase, a standard six-month regimen with an initial phase of four drugs resulted in negative sputum cultures at 30 months in $94 \% .{ }^{1}$ Furthermore, while levofloxacin has few adverse effects, irreversible hearing loss is a common finding in those treated with amikacin. ${ }^{2}$ Research itself is known to improve patient outcomes. Improved care rather than the addition of two further drugs to the standard treatment regimen for TB may have been responsible for the observed 'complete bacteriological cure at three months and radiological cure at the end of six months' that are reported in an unspecified number of patients from an, as yet unpublished, study.
G BOTHAMLEY

Department of respiratory medicine, Homerton University Hospital

M LIPMAN

Department of respiratory medicine, Royal Free Hospital

O M KON

Department of respiratory medicine, St. Mary's Hospital

\section{References}

1 Nunn AJ, Jindani A, Enarson DA, for the Study A investigators. Results at 30 months of a randomised trial of two 8 month regimens for the treatment of tuberculosis. Int J Tuberc Lung Dis 2011;15:741-5.

2 Sturdy A, Goodman A, Jose RJ et al. Multidrug-resistant tuberculosis (MDRTB) treatment in the UK: a study of injectable use and toxicity in practice. $J$ Antimicrob Chmeother 2011;66:1815-20.

\section{Influenza-related pneumonia}

Editor - In regard to the CME Respiratory Medicine article by Almond et al on influenza-related pneumonia (Clin Med February 2012 pp 67-70), I would like to correct a point on when to administer antiviral therapy.

It is stated in the article that based on Department of Health recommendations ${ }^{1}$ uncomplicated influenza infection should be treated with prompt commencement of antiviral therapy. However, more recent guidelines from the Health Protection Agency $^{2}$ state that generally in an uncomplicated presentation of influenza, treatment, other than symptomatic, is not required.

While it is correct that in the hospital setting all patients with influenza should be given antiviral drugs such as oseltamivir, in the community those patients with an uncomplicated presentation should only receive antiviral treatment if they have underlying health issues such as chronic heart, liver, pulmonary or renal disease.

Oseltamivir does have potential significant side effects such as nausea, vomiting and abdominal pain, and even reports of more serious adverse effects such as 